ORIGINAL ARTICLE

\title{
Radiographic Evaluation of the Hyoid Bone Position and Pharyngeal Airway Depth in Anteroposterior Dysplasia
}

\author{
Rajat Chauhan ${ }^{1}$, Dinesh Kumar Bagga ${ }^{2}$, Poonam Agrawal ${ }^{3}$, Hiten Kalra ${ }^{4}$, Prafful Kumar ${ }^{5}$, Anoop Singh ${ }^{6}$
}

\begin{abstract}
Introduction: The pharyngeal airway, tongue, and hyoid bone are correlated. The hyoid bone is connected to the pharynx, mandible, and cranium through muscles and ligaments and its position with respect to them determines the tongue posture and function. It also plays an important role in maintaining the airway and upright natural head position. Different skeletal patterns have different morphologies and shapes of the mandible, which might be affected by the position of the hyoid bone and the pharyngeal airway depth. This cephalometric study was conducted to relate the pharyngeal airway depth and the hyoid bone position of different skeletal patterns in sagittal dimension, and it determines and compares the linear parameters of the pharyngeal airway depth in Class I, II, and III malocclusion groups and anteroposterior, vertical, and angular positions of the hyoid bone positions in Class I, II, and III malocclusion groups.

Materials and methods: Lateral cephalometric radiographs selected for study were divided into three groups of 30 samples each based on ANB angle and $\beta$ angle into skeletal Class I, Class II, and Class III, respectively. Five linear measurements for the pharyngeal airway depth, four linear measurements for anteroposterior position, two linear measurements for vertical position, and one angular measurement for angular hyoid bone position were made.

Results: The pharyngeal airway depth was found to show no statistically significant difference between Class I and Class III malocclusions. The pharyngeal airway depth in Class II malocclusion was found to be statistically smaller at D1 as compared to that in Class I and Class III malocclusions, whereas it remained the same at the rest of the locations measured, i.e., D2, D3, D4, and D5. The Class III malocclusion group showed a significant statistical difference in the anteroposterior hyoid bone position at all levels (i.e., $\mathrm{H} 1, \mathrm{H} 2, \mathrm{H} 3$, and $\mathrm{H} 4$ ) as compared to the Class II malocclusion group, whereas it showed a statistically significant difference as compared to the Class I malocclusion group at $\mathrm{H} 1$ and $\mathrm{H}$ 3. The angular inclination of the hyoid bone in the Class II malocclusion group showed a statistically significant difference with the Class I and Class III malocclusion group but the Class III malocclusion group showed no significant difference statistically when compared to the Class I malocclusion group.

Conclusion: The pharyngeal airway depth in the Class II malocclusion group was also found to be narrower at the superiormost level measured at the posterior nasal spine from the posterior pharyngeal wall. The hyoid bone position was variable in angular and anteroposterior dimensions among different malocclusion groups.
\end{abstract}

Keywords: Anteroposterior dysplasia, Cephalometric radiographs, Hyoid bone, Pharyngeal airway, Skeleton malocclusion.

International Journal of Clinical Pediatric Dentistry (2019): 10.5005/jp-journals-10005-1601

\section{INTRODUCTION}

The patent pharyngeal airway is mandatory for the growth and development of the craniofacial region. ${ }^{1-4}$ The patent normal airway is maintained by the relationship of the pharynx with its associated structures, such as the tongue and hyoid bone. ${ }^{5}$ The hyoid bone is connected to the pharynx, mandible, and cranium through muscles and ligaments. ${ }^{6}$ The hyoid bone position with respect to the cranial base and the mandible determines the tongue posture and function, and it plays an important role in maintaining the airway and upright natural head position. The position of the hyoid bone is influenced by the position of the tongue, thus affecting the pharyngeal airway space. ${ }^{7}$ Thurow proposed that the anteroposterior position of the hyoid is maintained by the geniohyoid muscle due to which airway patency was maintained throughout the various movements of the craniofacial complex. ${ }^{8}$ The position of the hyoid bone on the genial tubercle level might increase the efficiency of the muscle in pulling the tongue forward and maintaining the airway. A more inferior position of the hyoid bone with lower tongue posture places the geniohyoid muscle at a mechanical disadvantage by its angulation. This may increase the mandibular load because of the need to elevate the tongue as well as to create a stronger opening force on the mandible, which can be of significance in the development and establishment of the dentofacial pattern and function. ${ }^{9}$
${ }^{1-6}$ Department of Orthodontics and Dentofacial Orthopedics, School of Dental Sciences, Sharda University, Greater Noida, Uttar Pradesh, India

Corresponding Author: Rajat Chauhan, Department of Orthodontics and Dentofacial Orthopedics, School of Dental Sciences, Sharda University, Greater Noida, Uttar Pradesh, India, Phone: +91 9711762547, e-mail: rajat18chauhan@gmail.com

How to cite this article: Chauhan R, Bagga DK, et al. Radiographic Evaluation of the Hyoid Bone Position and Pharyngeal Airway Depth in Anteroposterior Dysplasia. Int J Clin Pediatr Dent 2019;12(2):101-106.

Source of support: Nil

Conflict of interest: None

Assessment of sagittal dimension has been considered by applying the ANB angle in most of the studies, but it is affected by various factors and can often be misleading. When using the ANB angle, all those factors should be considered; this makes the interpretation of this angle much more complex than previously thought.

We have additionally applied the $\beta$-angle for selection of subjects along with the ANB angle as this measurement does not depend on cranial landmarks or the functional occlusal plane. ${ }^{10}$

Thus, it may be considered that different skeletal patterns have different morphologies and shapes of the mandible, which might 
be affected by the position of the hyoid bone and the pharyngeal airway depth. So, this study was conducted to relate the pharyngeal airway depth and hyoid bone position of different skeletal patterns in sagittal dimension.

\section{Materials and Methods}

This study was performed on a total of 90 lateral cephalometric radiographs, which were randomly selected from the records of Department of Orthodontics. Subjects included in this study had natural dentition and no craniofacial anomalies, syndromes, clefting, or symptoms or signs of dysfunction of the masticatory system. Standardized lateral cephalometric radiographs were taken in habitual occlusion with subject's head oriented parallel to the Frankfort horizontal plane.

The selected radiographs were divided into three groups of 30 samples each based on the ANB angle and $\beta$ angle into skeletal Class I, Class II, and Class III, respectively.

Class I: ANB angle, $0-4^{\circ}$ and $\beta$ angle $27-34^{\circ}$

Class II: ANB angle $>4^{\circ}$ and $\beta$ angle $<27^{\circ}$

Class III: ANB angle $<0^{\circ}$ and $\beta$ angle $>34^{\circ}$

The inclusion criteria of the selected samples of the respective groups had a normodivergent growth pattern based on a Frankfurt mandibular plane angle of 21-29.

All radiographs were traced by the same researcher and 21 landmarks were recorded (Fig. 1 and Table 1), which were used to calculate 11 linear and 1 angular measurements. There were five linear measurements for the pharyngeal airway depth (Fig. 2 and Table 2), four linear measurements for the anterior-posterior hyoid bone position, two linear measurements for the vertical hyoid bone position, and one angular measurement for the angular hyoid bone position (Fig. 3 and Table 3).

\section{Statistical Analysis}

Descriptive statistics composed of calculation of means, standard deviations, minimum and maximum value, and coefficient of variance. ANOVA was used to analyze comparison in three groups. The intergroup comparison of the pharyngeal airway depth and the hyoid bone position was performed using post hoc Tukey's test. A value of $p<0.05$ was considered statistically significant. All the data were analyzed by using SPSS version 21 .

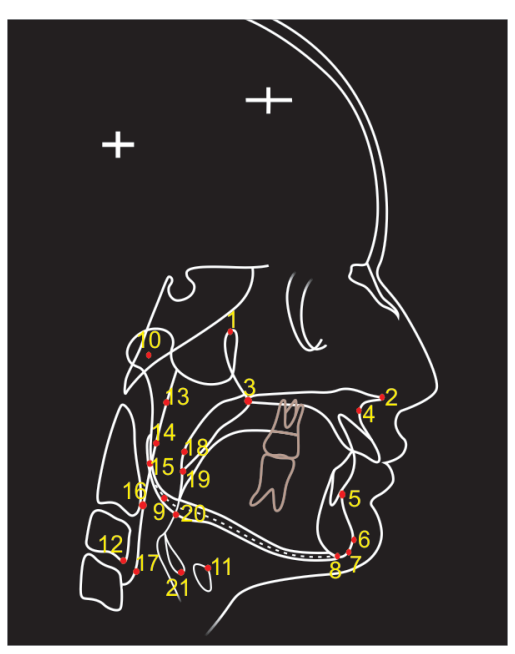

Fig. 1: Landmarks used in the study
Table 1: Landmarks used in the study

\begin{tabular}{|c|c|c|}
\hline & Landmark & Description \\
\hline 1 & Ptm & A bilateral teardrop-shaped area of radiolucency \\
\hline 2 & ANS & $\begin{array}{l}\text { Anterior tip of the sharp bony maxilla at the lower } \\
\text { margin of the anterior nasal opening }\end{array}$ \\
\hline 3 & PNS & The posterior spine of the palatine bone \\
\hline 4 & Point A & $\begin{array}{l}\text { The point at the deepest midline concavity on } \\
\text { the maxilla between the anterior nasal spine and } \\
\text { prosthion }\end{array}$ \\
\hline
\end{tabular}

5 Point B The point at the deepest midline concavity on the mandibular symphysis between infradentale and pogonion

6 Pog The most anterior point of the bony chin in the median plane

7 Gn The point located by taking the midpoint between the anterior and inferior points of the bony chin

$8 \mathrm{Me} \quad$ The lowest point on the symphyseal shadow of the mandible seen on the lateral cephalogram

9 Go The point on the curvature of the angle of the mandible located by bisecting the angle formed by lines tangent to the posterior ramus and the inferior border of the mandible

$10 \mathrm{C}$ The apparent axis of the condyle

$11 \mathrm{H}$ The anterosuperior point on the hyoid bone

12 C3 The most anteroinferior point of the third vertebra

13 SPU The point on the posterior pharyngeal wall along the palatal plane

14 SPM The point on the posterior pharyngeal wall where the distance to SAM is the shortest

15 SPML The point on the posterior pharyngeal wall where the distance to $\mathrm{P}$ is the shortest

$16 \mathrm{SPL}$ The point on the posterior pharyngeal wall where the distance to SAL is the shortest

17 SPLL The point on the posterior pharyngeal wall where the distance to $\mathrm{E}$ is the shortest

18 SAM The point on the soft palate where the postpalatal airway is narrowest

$19 \mathrm{P} \quad$ The tip of the soft palate

20 SAL The point on the tongue along the mandibular lower border

21 E Epiglottis

\section{Results}

The pharyngeal airway depth was found to show no statistically significant difference between Class I and Class III malocclusions. The pharyngeal airway depth in Class II malocclusion was found to be statistically significantly smaller at D1 as compared to that of Class I and Class III malocclusion, whereas it remained the same at the rest of the locations measured, i.e., D2, D3, D4, and D5 (Table 4).

The anteroposterior hyoid bone position in the Class II malocclusion group showed statistically insignificant difference as compared to that of the Class I malocclusion group at all levels (i.e., $\mathrm{H1}, \mathrm{H} 2, \mathrm{H} 3$, and H4). The Class III malocclusion group showed statistically significant difference in the anteroposterior hyoid bone position at all levels (i.e., H1, H2, H3, and H4) as compared to the Class II malocclusion group, whereas it showed statistically significant difference as compared to the Class I malocclusion group 


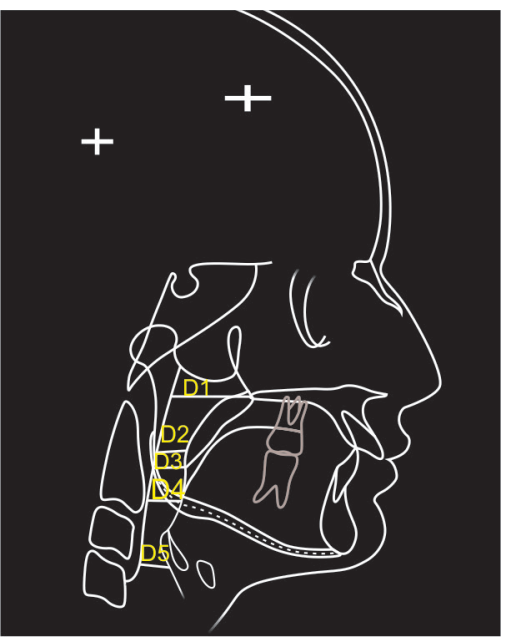

Fig. 2: Measurements of pharyngeal airway depth (in $\mathrm{mm}$ )

Table 2: Measurements of pharyngeal airway depth (in $\mathrm{mm}$ )

D1 Linear distance between the posterior spine of the palatine palatal plane

D2 Linear distance between the posterior pharyngeal wall and the point on the soft palate where the postpalatal airway is narrowest

D3 Linear distance between the posterior pharyngeal wall and the tip of the soft palate where the distance is shortest

D4 Linear distance between the posterior pharyngeal wall and the point on the tongue along the mandibular lower border where the distance is shortest

D5 Linear distance between the posterior pharyngeal wall and Epiglottis where the distance is shortest

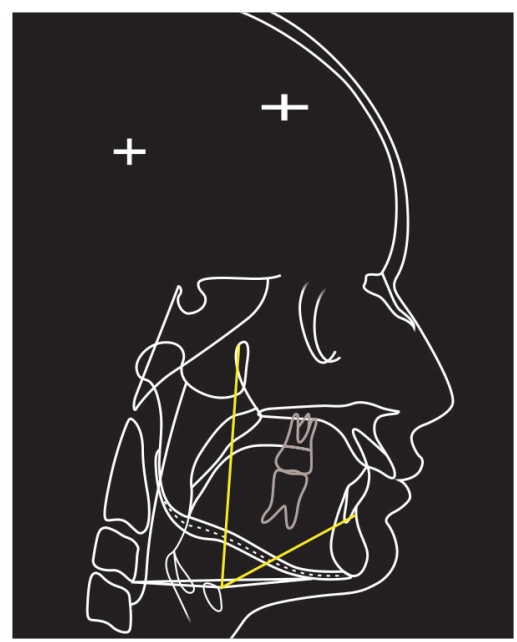

Fig. 3: Measurements of hyoid bone position (in $\mathrm{mm}$ )

at $\mathrm{H} 1$ and $\mathrm{H} 3$ remaining the same at the rest of the locations, i.e., $\mathrm{H} 2$, and $\mathrm{H} 4$ (Table 5).

There was no statistically significant difference in the vertical hyoid bone position in Class I, Class II, and Class III malocclusion groups (Table 6).

The angular inclination of the hyoid bone in the Class II malocclusion group showed statistically significant difference with Class I and Class III malocclusion groups but the Class III
Table 3: Measurements of hyoid bone position (in $\mathrm{mm}$ )

$\mathrm{H} 1 \quad$ Horizontal linear distance between the anterosuperior point on hyoid bone and Menton

$\mathrm{H} 2$ Horizontal linear distance between the anterosuperior point on the hyoid bone and most anteroinferior point of the third cervical verterbra

H3 Horizontal linear distance between the anterosuperior point on hyoid bone and Point B

H4 Horizontal linear distance between the anterosuperior point on the hyoid bone and epiglottis

H5 Vertical distance between the anterosuperior point on the hyoid bone and Ptm

H6 The vertical distance from anterosuperior point on the hyoid bone to the plane formed by most anteroinferior point of the third cervical verterbra and Menton

H7 The angle formed by epiglottis, anterosuperior point on the hyoid bone and Point $B$

malocclusion group showed no statistically significant difference when compared to the Class I malocclusion group (Table 7).

\section{Discussion}

A normal nasal airway is dependent on sufficient anatomical dimensions of the airway and also on its relationship with the pharynx and its associated structures such as the tongue and hyoid bone. The position of the pharynx changes with the change in position of the hyoid bone and the tongue, whereas the mandibular position brings about variations in the hyoid bone and tongue. Thus, different skeletal patterns would lead to changes indirectly in the pharyngeal airway space. The present cephalometric study determines and compares the linear parameters of the pharyngeal airway depth in the Class I, II, and III malocclusion groups and the anteroposterior, vertical, and angular position of the hyoid bone in the Class I, II, and III malocclusion groups.

The pharyngeal airway depth was comparable in the Class I and Class III malocclusion groups except the Class II malocclusion group, which showed a narrower pharyngeal depth at the superiormost level measured at the posterior nasal spine from the posterior pharyngeal wall as compared to that in the Class I and Class III malocclusion groups but remained the same at the inferior part of the pharyngeal airway dimension. These findings were in agreement with a study done by Kerr, who reported a smaller nasopharyngeal airway area in Class II malocclusion than normal occlusion. ${ }^{12}$ On the other hand, the results were in contrast to the study done by Zhe Zhong, et al., which found no significant difference in the superior part of the upper airway dimension at PNS and soft palate in Class I, Class II, and Class III malocclusion groups but the inferior part of the upper airway dimension (i.e., at the level of tip of the uvula, point of intersection of base of the tongue, and extension of line B-Go and the posteroinferior point on the base of the tongue) noted significant decrease from Class III to Class I to Class II malocclusion groups in the normodivergent facial pattern.

Alves et al. reported an increased pharyngeal airway depth in the Class III malocclusion group as compared to that of the Class I malocclusion group at the uvula in children (age 8-10 years), which is in contrast to the findings of our study as there was no significant difference in the pharyngeal airway depth between Class I and Class III malocclusion groups in our sample. ${ }^{13,14}$ The study conducted by Freitas et al. is also not in agreement with the 
Table 4: Measurement of pharyngeal airway depth and results of statistical analysis of measurements for pharyngeal airway depth between three groups (post hoc Tukey's test)

\begin{tabular}{|c|c|c|c|c|c|c|c|c|}
\hline Parameter & Group & Mean $(\mathrm{mm})$ & $S D(m m)$ & $\begin{array}{l}\text { Minimum } \\
(\mathrm{mm})\end{array}$ & $\begin{array}{l}\text { Maximum } \\
(\mathrm{mm})\end{array}$ & CV & $p$ value & $\begin{array}{l}\text { Post hoc pairwise comparison } \\
\text { by Tukey's test }\end{array}$ \\
\hline \multirow[t]{3}{*}{ D1 } & $\mathrm{ClI}$ & 28.55 & 5.63 & 22 & 39 & 19.71 & $0.007, \mathrm{~S}$ & $1 \times 2-0.046$ \\
\hline & $\mathrm{Cl} I \mathrm{I}$ & 25.98 & 1.85 & 23 & 30 & 7.1 & & $1 \times 3-0.796$ \\
\hline & $\mathrm{Cl} \mathrm{III}$ & 29.23 & 3.94 & 24 & 35 & 13.47 & & $2 \times 3-0.008$ \\
\hline \multirow[t]{3}{*}{ D2 } & $\mathrm{Cll}$ & 10.32 & 0.97 & 8.5 & 12 & 9.39 & $0.735, \mathrm{NS}$ & $1 \times 2-0.813$ \\
\hline & $\mathrm{Cl} I \mathrm{I}$ & 9.97 & 3.39 & 4 & 13.5 & 34 & & $1 \times 3-0.992$ \\
\hline & $\mathrm{Cl}$ III & 10.38 & 1.49 & 7 & 12 & 14.35 & & $2 \times 3-0.746$ \\
\hline \multirow[t]{3}{*}{ D3 } & $\mathrm{Cll}$ & 11.18 & 1.48 & 8 & 13 & 13.23 & 0.901, NS & $1 \times 2-0.992$ \\
\hline & $\mathrm{Cl} I \mathrm{I}$ & 11.08 & 3.21 & 5 & 15.5 & 28.97 & & $1 \times 3-0.960$ \\
\hline & $\mathrm{Cl} \mathrm{III}$ & 11.33 & 1.04 & 9.5 & 14 & 9.17 & & $2 \times 3-0.892$ \\
\hline \multirow[t]{3}{*}{ D4 } & $\mathrm{Cll}$ & 12.37 & 1.65 & 8 & 14 & 13.33 & 0.260, NS & $1 \times 2-0.892$ \\
\hline & $\mathrm{Cl} I \mathrm{I}$ & 12.08 & 3.50 & 5 & 16 & 28.97 & & $1 \times 3-0.486$ \\
\hline & $\mathrm{Cl} \mathrm{III}$ & 13.08 & 1.57 & 11 & 17 & 12 & & $2 \times 3-0.249$ \\
\hline \multirow[t]{3}{*}{ D5 } & $\mathrm{Cll}$ & 17.90 & 3.66 & 14 & 26 & 20.44 & $0.313, \mathrm{NS}$ & $1 \times 2-0.508$ \\
\hline & $\mathrm{Cl} I \mathrm{I}$ & 16.75 & 5.68 & 2 & 27 & 33.9 & & $1 \times 3-0.933$ \\
\hline & $\mathrm{Cl}$ III & 18.27 & 1.49 & 16 & 22 & 8.15 & & $2 \times 3-0.310$ \\
\hline
\end{tabular}

Table 5: Anteroposterior measurement of hyoid bone position and results of statistical analysis of measurements for hyoid bone position between three groups (post hoc Tukey's test)

\begin{tabular}{|c|c|c|c|c|c|c|c|c|}
\hline Parameter & Group & Mean $(\mathrm{mm})$ & $S D$ & $\operatorname{Min}(\mathrm{mm})$ & $\operatorname{Max}(\mathrm{mm})$ & $C_{V}$ & pvalue & Post hoc Tukey's test \\
\hline \multirow[t]{3}{*}{$\mathrm{H} 1$} & $\mathrm{ClI}$ & 39.67 & 3.15 & 35 & 46 & 7.94 & $<0.001, \mathrm{~S}$ & $1 \times 2-0.213$ \\
\hline & $\mathrm{Cl} I \mathrm{I}$ & 38.22 & 3.19 & 33 & 46 & 8.34 & & $1 \times 3-<0.001$ \\
\hline & $\mathrm{Cl}$ III & 47.57 & 3.59 & 42 & 53 & 7.54 & & $2 \times 3-<0.001$ \\
\hline \multirow[t]{3}{*}{$\mathrm{H} 2$} & $\mathrm{Cll}$ & 37.95 & 4.06 & 32 & 47 & 10.69 & $0.033, \mathrm{~S}$ & $1 \times 2-0.094$ \\
\hline & $\mathrm{Cl} I \mathrm{I}$ & 35.18 & 6.51 & 28 & 48 & 18.50 & & $1 \times 3-0.933$ \\
\hline & $\mathrm{Cl} I \mathrm{II}$ & 38.42 & 4.33 & 29 & 44 & 11.27 & & $2 \times 3-0.041$ \\
\hline \multirow[t]{3}{*}{$\mathrm{H} 3$} & $\mathrm{Cll}$ & 51.00 & 4.89 & 44 & 62 & 9.58 & $<0.001, \mathrm{~S}$ & $1 \times 2-0.435$ \\
\hline & $\mathrm{Cl} I \mathrm{I}$ & 49.62 & 2.77 & 45.5 & 54.5 & 5.58 & & $1 \times 3-<0.001$ \\
\hline & $\mathrm{Cl} I \mathrm{II}$ & 57.27 & 4.98 & 51 & 64 & 8.69 & & $2 \times 3-<0.001$ \\
\hline \multirow[t]{3}{*}{$\mathrm{H} 4$} & $\mathrm{ClI}$ & 14.82 & 4.31 & 10 & 26 & 29.08 & $0.049, \mathrm{~S}$ & $1 \times 2-0.216$ \\
\hline & $\mathrm{Cl} I \mathrm{I}$ & 13.28 & 2.03 & 11 & 17 & 15.28 & & $1 \times 3-0.734$ \\
\hline & $\mathrm{Cl} I \mathrm{II}$ & 15.50 & 3.81 & 10 & 23 & 24.58 & & $2 \times 3-0.044$ \\
\hline
\end{tabular}

Table 6: Vertical measurement of the hyoid bone position and results of statistical analysis of measurements for the hyoid bone position between the three groups (post hoc Tukey's test)

\begin{tabular}{|c|c|c|c|c|c|c|c|c|}
\hline Parameter & Group & Mean $(m m)$ & $S D$ & $\operatorname{Min}(m m)$ & $\operatorname{Max}(\mathrm{mm})$ & $C v$ & $p$ value & Post hoc Tukey's test \\
\hline \multirow[t]{3}{*}{ H5 } & $\mathrm{Cll}$ & 87.30 & 11.64 & 78 & 112 & 13.33 & $0.041, \mathrm{~S}$ & $1 \times 2-0.996$ \\
\hline & $\mathrm{Cl} I \mathrm{I}$ & 87.12 & 6.13 & 82 & 98 & 7.03 & & $1 \times 3-0.079$ \\
\hline & $\mathrm{Cl}$ III & 92.25 & 7.61 & 78 & 108 & 8.24 & & $2 \times 3-0.066$ \\
\hline \multirow[t]{3}{*}{$\mathrm{H} 6$} & $\mathrm{Cll}$ & 4.90 & 3.58 & 1 & 14 & 73.06 & $0.840, \mathrm{NS}$ & $1 \times 2-0.991$ \\
\hline & $\mathrm{Cl} I \mathrm{I}$ & 4.82 & 1.65 & 2 & 8 & 34.23 & & $1 \times 3-0.891$ \\
\hline & $\mathrm{Cl}$ III & 4.53 & 1.87 & 1 & 7 & 41.28 & & $2 \times 3-0.901$ \\
\hline
\end{tabular}

present study as they found insignificant differences in the upper airway dimension among Class I and Class II malocclusion subjects with a normodivergent growth pattern. ${ }^{15}$

Ravinarayana Reddy et al. conducted a study in Class I, Class II, and Class III malocclusion groups with different skeletal patterns and reported that in Class III malocclusion with a normal skeletal pattern, upper and lower pharyngeal airway depths were significantly more than that in the Class I malocclusion group with a normal skeletal pattern, which was not in agreement with our study. Reddy et al. also concluded that in the Class I and Class II malocclusion groups with a normal skeletal pattern, there was no statistically significant difference in the upper and lower pharyngeal airway depths and there was statistically significant difference in Class II and Class III malocclusion groups with a normal skeletal pattern in the lower pharyngeal airway depth, which was similar to our result. ${ }^{16}$

In the present study, the anteroposterior position of the hyoid bone was comparable in the Class I and Class II malocclusion groups. The anteroposterior position of the hyoid bone in the Class III malocclusion group was found to have the same distance 
Table 7: Angular inclination measurement of the hyoid bone position and results of statistical analysis of measurements for hyoid bone position between the three groups (post hoc Tukey's test)

\begin{tabular}{lllllllll}
\hline Parameter & Group & Mean $(\mathrm{mm})$ & $\mathrm{SD}$ & Min $(\mathrm{mm})$ & Max $(\mathrm{mm})$ & CV & p value & Post hoc Tukey's test \\
\hline $\mathrm{H} 7$ & ClI & 155.20 & 8.05 & 143 & 172 & 5.18 & $0.002, \mathrm{~S}$ & $1 \times 2-0.016$ \\
& Cl II & 149.30 & 5.56 & 143 & 163 & 3.73 & $1 \times 3-0.792$ \\
& Cl III & 156.57 & 10.09 & 130 & 165 & 6.44 & $2 \times 3-0.002$ \\
\hline
\end{tabular}

from the third cervical verterbra and epiglottis as that in the Class I malocclusion group, whereas distance was increased when measured from Point $B$ and menton.

The anteroposterior position of the hyoid bone was found to be anterior in the Class III malocclusion group than that in the Class II malocclusion group, showing increased distances between the third cervical verterbra and epiglottis posteriorly as well as between menton and Point B anteriorly.

The vertical position of the hyoid bone remained unchanged irrespective of the malocclusion groups. The angular position of the hyoid bone was comparable in the Class I and Class III malocclusion groups. The angular position of the hyoid bone in Class II malocclusion group was found to be at lesser angulation than the Class I and Class III malocclusion groups.

This result was similar to a study by Adamidis and Spyropoulos who reported a significant difference in the anteroposterior position of the hyoid bone between Class I and Class III malocclusions as the hyoid bone was laid more anteriorly in Class III than in Class I, possibly due to the muscular attachment to the hyoid bone and the mandible moving the hyoid forward in the sagittal plane. They also concluded that the vertical position remained the same in Class I and Class III malocclusions, which was similar to our study. ${ }^{17}$

Kuroda et al. conducted a study to determine the relationship between the hyoid bone, skull, and mandible by using lateral cephalograms. They concluded that in Class II samples the hyoid bone was located backward and in the Class III samples the hyoid bone was located forward in comparison with the control group. In our study, the hyoid bone was located at the same position as in Classes I, II, and III malocclusion groups except that the distance was increased when measured from Point $B$ and menton to hyoidale in the Class III malocclusion group. ${ }^{18}$

Chauhan et al. conducted a study to compare the pharyngeal airway dimension, tongue, and hyoid bone position in Class I and Class II Division 1 and reported that there was no statistically significant difference in the pharyngeal airway anterior-posterior dimension and also in the position and relationship of the hyoid bone and tongue between Class I and Class II Division I, which was similar to our result. ${ }^{6}$

Our study lacks in the exclusion of samples with different types of growth pattern as we have included only normodivergent subjects but it combines the measurement of pharyngeal airway depth and hyoid bone positions in different groups of anteroposterior dysplasia having a normodivergent growth pattern, which provides an assessment tool for future comparative studies.

\section{ConCLUSION}

The pharyngeal airway depth and angular position of hyoid bone were comparable in Class I and Class III malocclusion groups. The pharyngeal airway depth in the Class II malocclusion group was also found to be the same as that in the Class I and Class III malocclusion groups except at the superiormost level measured at the posterior nasal spine from the posterior pharyngeal wall where it was narrower in the Class II malocclusion group.

The angular position of the hyoid bone in the Class II malocclusion group was found to be at lesser angulation than the Class I and Class III malocclusion groups.

The vertical position of the hyoid bone remained unchanged irrespective of the malocclusion groups. The anteroposterior position of the hyoid bone was comparable in Class I and Class II malocclusion groups.

The anteroposterior position of the hyoid bone in the Class III malocclusion group was found to be the same as that in the Class I malocclusion group when measured from the third cervical verterbra and epiglottis posteriorly whereas it was found to be increased in the Class III malocclusion group compared to that in the Class I malocclusion group when measured from hyoidale to Point B and menton.

The anteroposterior position of the hyoid bone was found to be anterior in the Class III malocclusion group compared to that in the Class II malocclusion group, showing increased distances between the third cervical verterbra and epiglottis posteriorly as well as between menton and Point B anteriorly.

\section{References}

1. Dunn GF, Green LJ, et al. Relationships between variation of mandibular morphology and variation of nasopharyngeal airway size in monozygotic twins. Angle Orthod 1973;43:129-135. DOI: 10.1043/0003-3219(1973)043<0129:RBVOMM>2.0.CO;2.

2. MCNamara JA Jr. Influence of respiratory pattern on craniofacial growth. Angle Orthod 1981;51:269-300. DOI: 10.1043/0003-3219(1981)051<0269:IORPOC>2.0.CO;2.

3. Ceylan I, Oktay H. A study on the pharyngeal size in different skeletal patterns. Am J Orthod Dentofacial Orthop 1995;108:69-75.

4. Solow B, Siersbaek S, et al. Airway adequacy, head posture, and craniofacial morphology. Am J Orthod 1984;86:214-223.

5. Sheng CM, Lin LH, et al. Developmental changes in pharyngeal airway depth and hyoid bone position from childhood to adulthood. Angle Orthod 2009;79:484-490. DOI: 10.2319/062308-328.1.

6. Chauhan A, Autar R, et al. Comparison of pharyngeal airway dimension, tongue and hyoid bone position based on ANB angle. Natl J Maxillofac Surg 2015;6(1):42-51. DOI: 10.4103/0975-5950.168237.

7. Jenaa AK, Duggal R. Hyoid bone position in subjects with different vertical jaw dysplasias. Angle Orthod 2011;81:81-85. DOI: 10.2319/092208-491.1.

8. Kjellberg H. Juvenile chronic arthritis. Dentofacial morphology, growth, mandibular function and orthodontic treatment. Swed Dent J Suppl 1995;109:1-56.

9. Stratemann S, Huang JC, et al. Three-dimensional analysis of the airway with cone-beam computed tomography. Am J Orthod Dentofacial Orthop 2011;140:607-615. DOI: 10.1016/j.ajodo.2016.06.049.

10. Baik CY, Ververidou M. A new approach of assessing sagittal discrepancies: the beta angle. Am J Orthod Dentofacial Orthop 2004;126:100-105. DOI: 10.1016/S0889540604002483.

11. Zhonga Z, Tangb Z, et al. A comparison study of upper airway among different skeletal craniofacial patterns in nonsnoring Chinese children. Angle Orthod 2010;80:267-274. DOI: 10.2319/061917-399.12. 
12. Kerr WJ. The nasopharynx, face height and overbite. Angle Orthod 1985;55(1):31-36. DOI: 10.1043/0003-3219(1985)055<0031:TNFHAO> 2.0.CO;2.

13. Alves Jr M, Franzotti ES, et al. Evaluation of pharyngeal airway space amongst different skeletal patterns. Int J Oral Maxillofac Surg 2012;41:814-819. DOI: 10.1016/j.ijom.2012.01.015.

14. Ang PK, Sandham A, et al. Craniofacial morphology and head posture in Chinese subjects with obstructive sleep apnea. Semin Orthod 2004;10:90-96.

15. Freitas MR, Alcazar NMP, et al. Upper and lower pharyngeal airways in subjects with class I and class II malocclusions and different growth patterns. Am J Orthod Dentofacial Orthop 2006;130:742-745. DOI: 10.1016/j.ajodo.2005.01.033.

16. Reddy R, Chunduri R, et al. Upper and Lower Pharyngeal Airways In Subjects With Skeletal Class-I, Class-II \& Class-III Malocclusions And Different Growth Patterns - A Cephalometric Study. Int J Contemp Dent 2011;2(5):12-18.

17. Adamidis IP, Meropi N. Spyropoulos hyoid bone position orientation in class I and class III malocclusions. Am J Orthod Dentofac Orthop 1992;101:308-312. DOI: 10.1016/S0889-5406(05)80323-3.

18. Kuroda T, Nunota E, et al. A roentgenocephalometric study on the position of the hyoid bone. Bull Tokyo Med Dent Univ 1966;13:227-243. 